\title{
Improving Trading Systems Using the RSI Financial Indicator and Neural Networks
}

\author{
Alejandro Rodríguez-González ${ }^{1}$, Fernando Guldrís-Iglesias ${ }^{1}$, \\ Ricardo Colomo-Palacios ${ }^{1}$, Juan Miguel Gomez-Berbis ${ }^{1}$, Enrique Jimenez-Domingo ${ }^{1}$, \\ Giner Alor-Hernandez ${ }^{2}$, Rubén Posada-Gomez ${ }^{2}$, and Guillermo Cortes-Robles ${ }^{2}$ \\ ${ }^{1}$ Universidad Carlos III de Madrid, Av. Universidad 30, Leganés, 28918, Madrid, Spain \\ \{alejandro.rodriguez, fernando.guldris,ricardo.colomo, \\ juanmiguel.gomez \}ac3m.es \\ ${ }^{2}$ Division of Research and Postgraduate Studies, \\ Instituto Tecnológico de Orizaba, México \\ \{galor, rposada, gcortes\} @itorizaba.edu.mx
}

\begin{abstract}
Trading and Stock Behavioral Analysis Systems require efficient Artificial Intelligence techniques for analyzing Large Financial Datasets (LFD) and have become in the current economic landscape a significant challenge for multi-disciplinary research. Particularly, Trading-oriented Decision Support Systems based on the Chartist or Technical Analysis Relative Strength Indicator (RSI) have been published and used worldwide. However, its combination with Neural Networks as a branch of computational intelligence which can outperform previous results remain a relevant approach which has not deserved enough attention. In this paper, we present the Chartist Analysis Platform for Trading (CAST, in short) platform, a proof-of-concept architecture and implementation of a Trading Decision Support System based on the RSI and FeedForward Neural Networks (FFNN). CAST provides a set of relatively more accurate financial decisions yielded by the combination of Artificial Intelligence techniques to the RSI calculation and a more precise and improved upshot obtained from feed-forward algorithms application to stock value datasets.
\end{abstract}

Keywords: Neural Networks, RSI Financial Indicator.

\section{Introduction}

There has been growing interest in Trading Decision Support Systems in recent years. Forecasting the price movements in stock markets has been a major challenge for common investors, businesses, brokers and speculators. The stock market is considered as a high complex and dynamic system with noisy, non-stationary and chaotic data series [1], and hence, difficult to forecast [2]. However, despite its volatibility, it is not entirely random [3], instead, it is nonlinear and dynamic [4] or highly complicated and volatile [5]. Stock movement is affected by the mixture of two types of factors [6]: determinant (e.g. gradual strength change between buying side and selling side) and random (e.g. emergent affairs or daily operation variations). 
Wen et al. [1], argues that the study of the stock market behavior is a hot topic, because if it is successful, the result will yield fruitful rewards. Thus, it is obvious that predicting stock market movement is the long cherished desire of investors, speculators, and industries. However, this market is extremely hard to model with any reasonable accuracy [2]. Prediction of stock price variation is a very difficult task and the price movement behaves more like a random walk and time varying [7].

However, despite the referred complexity, many factors, including macroeconomic variables and stock market technical indicators, have been proven to have a certain level of forecast capability on stock market during a certain period of time. One of the tools for the financial practice is technical analysis, also known as "charting". According to Leigh et al. [8] Charles Dow developed the original Dow Theory for technical analysis in 1884 revisited by Edwards and Magee [9] more than a century before. Technical analysis studies the historical data surrounding price and volume movements of the stock by using charts as the primary tool to forecast future price movements. In recent years, and in spite of several critics [10], technical analysis has proven to be powerful for evaluating stock prices and is widely accepted among financial economists and brokerage firms.

The paper consists of five sections and is structured as follows. Section 2 surveys the relevant literature about technical analysis and its intersection with soft computing. Section 3 discusses the main features of CAST including conceptual model, algorithmic and architecture. Section 4 describes the evaluation of the tool performed. Finally, the paper ends with a discussion of research findings, limitations and concluding remarks.

\section{Related Works}

Stock price prediction using soft intelligence methods is not new. To solve the nonlinear problem and improve stock price evaluation, many researchers have focused on Technical Analysis by using advanced mathematics based techniques [11]. Along with the development of Artificial Intelligence, more researchers have tried to build automatic decision-making systems to predict stock market [12]. Soft computing techniques such as fuzzy logic, neural networks, and probabilistic reasoning draw most attention because of their abilities to handle uncertainty and noise in stock market.

White [13] was the first to use neural networks for market forecasting in the late 80s. In the early 90s Kimoto, Asakawa, Yoda, and Takeoka [14] used several learning algorithms and prediction methods for developing a prediction system for the Tokyo Stock Exchange Prices Index. Trippi and DeSieno [15] combined the outputs of individual networks using boolean operators to produce a set of composite rules. Other approaches can be found in various works from that decade using artificial neural networks [16] or computational intelligence [17].

As stated before, CAST (Chartist Analysis System for Trading) is based on the use of an improved version of RSI, one of the leading technical analysis indexes. RSI as a part of diverse calculations and formulas is commonly present in the soft computing research [18]. However, using soft computing methods in getting iRSI calculations is 
a research task with no presence in the literature. Seeking this new research scenario in this work is proposed CAST, a system that uses generalized feedforward neural network to perform RSI improved calculations.

\section{The CAST System}

The idea of the system developed is to create a trading system based on technical or chartist analysis. Concretely, the main idea is to use one of the most used financial indicators, namely RSI. RSI is a financial technical analysis momentum oscillator measuring the velocity and magnitude of directional price movement by comparing upward and downward close-to-close movements. Momentum measures the rate of the rise or fall in stock price. Is the momentum increasing in the "up" direction, or is the momentum increasing in the "down" direction. Wilder [19] established that the more accurate value for $\mathrm{N}$ to calculate the best RSI is 14 because it was the half of the lunar cycle. However, depending on the market, the company and other factors, value 14 is not always the best value to calculate RSI. For this reason, in this paper is described a system capable of predict RSI values for a concrete market instead for a concrete company. The main idea is trying to predict the market behavior, concretely RSI behavior (as a collection of companies) and particularize for a concrete company using some correction factors. The system is divided in the following modules: neural network module, trading system module, RSI manager and generator module and heuristic module.

\subsection{Neural Network Module}

The Neural Network Module is the responsible of provide the RSI values that will be used to decide if an investor should invest in a certain company. The network used is a generalized feed-forward network [20]. The advantage of the generalized Feed Forward (FF) network is the ability to project activities forward by bypassing layers. The result is that the training of the layers closer to the input becomes much more efficient. Hereafter are showed the configuration of the network:

In first place, the input values of the network:

Table 1. Neural Network Input Values

\begin{tabular}{|ll|}
\hline \multicolumn{1}{|l|}{ Input Values } & Explanation \\
ValueX35 Action & Value of the market for a concrete day. \\
\hline RSI(9) & Calculus of RSI value using N =9. \\
\hline RSI(14) & Calculus of RSI value using N =14. \\
\hline RSI(30) & Calculus of RSI value using N = 30. \\
\hline RSI Optimal & Calculus of optimal RSI using Heuristic \\
\hline
\end{tabular}

The topology of the network used was divided in an input layer, one hidden layer and an output layer. The next table shows the neurons set to each layer. 
Table 2. Neural Network Configuration

\begin{tabular}{|c|c|c|c|}
\hline Layer & $\begin{array}{l}\text { Input } \\
\text { Neurons }\end{array}$ & \begin{tabular}{|l|} 
Output \\
Neurons
\end{tabular} & Activation Function \\
\hline Input & 5 & 15 & $\begin{array}{lllll}\text { Laguarre } & (3 & \text { Taps, } & 1 & \text { Tap } \\
\text { Delay) }\end{array}$ \\
\hline Hidden & 15 & 10 & TanH \\
\hline Output & 10 & 1 & Bias \\
\hline
\end{tabular}

The momentum value associated to the layers of the networks was set to 0.7. Following are showed the training values used in the network:

Table 3. Neural Network training set

\begin{tabular}{|c|c|c|}
\hline Number of input data & Cross Validation & Training \\
\hline 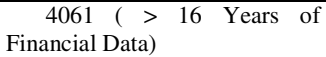 & Data & $\begin{array}{l}10 \% \text { of } \\
\text { Data }\end{array}$ \\
\hline
\end{tabular}

The number of epochs set in order to train the network was specified to 10,000 epochs establishing that the network must stop learning after 500 epochs without improvement in cross-validation error.

\subsection{Trading System Module}

This module analyzes the result given by neural network module. When a particular query is formulated to the system, it takes the actual values of the market and builds a query to the neural network. It is important to stand out that in this case the predictions made by the system affect only to the IBEX35 (Spanish) market and not to a concrete company. However, in the heuristic section, some correction values will be shown in order to see that is possible to adapt the heuristic method to make predictions directly to the companies. The analysis made by the trading system is simple. It takes the value given by the neural network (RSI) and compares it with two extreme values.

- If RSI value is higher than 70 the decision that trading system will return is a sell signal. This value can be adapted and in some cases the value will be set to 65 instead of 70 .

- If RSI value is lower than 30 the decision that trading system will return is a buy signal. This value can be adapted and in some cases the value will be set to 35 instead of 30 .

\subsection{RSI Manager and Generator Module}

The RSI Manager and generator is the module in charge of manages and generates the RSI Values. This module calculates RSI(N) values where $N \in[5,35]$ and is used to train the network or to query it. However, there is another RSI estimation that must be done: Optimal RSI. There are two ways to calculate the Optimal RSI. 
- Optimal RSI for Market (IBEX35) prediction: The calculus is provided by heuristic function (see next section).

- Optimal RSI for Company (IBEX35) prediction: In the context of company prediction Optimal RSI calculus is done taking into account the values that actions has in all the period without any modification, so, partial calculated RSIs are not stored and always is used the RSI of the day before and the actual day without taking into account previous RSIs.

\subsection{Heuristic Module}

The heuristic module is in charge of managing the different formulas that provide the heuristic used to generate the optimal values for RSI indicator. As was commented in the previous section exists more than one way to calculate the Optimal RSI value.

\section{Optimal RSI for Market (IBEX35) prediction:}

The formula of this heuristic is the next:

$$
R S I=C 1+C 2 * I B E X 35+C 3
$$

Where:

- IBEX35 represents the current value of the market on the actual date.

- $\quad \mathrm{C} 1$ is a correction parameter set to -206.1103731082200000

- $\mathrm{C} 2$ is a correction parameter set to 0.0213265642264200

- $\quad \mathrm{C} 3$ is a correction parameter set to 0.9947712774802800

The formula has been obtained using statistical techniques applied to the variables involved in the process. Concretely, a linear regression to relate the RSI values with IBEX35 stock market close values has been done. A total of 30 linear regressions were done to be able to collect possible values of RSI in function of the number of days. Each of these regressions used a different value of RSI when calculate itself with different time intervals. Concretely, it was permitted that the number of days used in the calculation could move between 5 and 35 . The reason is to collect all the possible values used, because it is not normal that analysts use periods out of this range.

Each of the linear regressions calculated include a parameter called AR(1) to improve the specification of the model. Once results have been obtained, the models that did not fulfill the next requisites were discarded:

- Some of the independent variables is not statistical meaningful for explain the behavior of dependent variable.

- The probability of whole nullity of the model is zero.

- The goodness of fit is not enough good. In this sense, are considerate as no valid the models that cannot explain a $75 \%$ of the variation of the dependent variable. 
- The model presents heteroscedasticity.

- The model presents autocorrelation.

Finally, in function of the mentioned criterions, has been discarded the models that include the RSI calculated with intervals of days equals to 5, 10, 12, 28, 30 and 35 . With the rest of the models we proceed to calculate a unique equation that is its arithmetic mean, in order that the resulted heuristic is the more representative value of all the valid calculated models.

\section{Optimal RSI for Company (IBEX35) prediction:}

The formula of this heuristic is the next (explained in several steps):

$$
\begin{aligned}
& \text { rsiToday }=C 1+(C 2 * v M T)+C 3 \\
& \text { rsiYday }=C 1+(C 2 * v M Y)+C 3 \\
& \text { restRSI }=\text { rsiToday }- \text { rsiYday } \\
& \text { growthRate Company }=(v C T-v C Y) / v C Y \\
& \text { growthRate Market }=(v M T-v M Y) / v M Y \\
& R S I=\text { rsiYday }+\left(\text { restRSI } * \frac{\text { growthRateCompany }}{\text { growthRateMarket }}\right)
\end{aligned}
$$

Where:

- vMT represents the value of the market today.

- vMY represents the value of the market yesterday.

- $\quad \mathrm{vCT}$ represents the value of the company today.

- $\mathrm{vCY}$ represents the value of the company yesterday.

- $\mathrm{C} 1, \mathrm{C} 2$ and $\mathrm{C} 3$ are the same parameters with the same values.

\section{Evaluation}

The evaluation of the system consists in two parts. In the first place, the evaluation of the neural network and how they are able to predict will be evaluated. Particularly, in this evaluation the best configuration has been chosen for a he neural network set up to calculate better predictions using the iRSI. The second part consists of a definite query to the neural networks with a certain number of values and checks the signals that RSI value shots (buy or sell). The aim is to find out the accuracy of CAST.

\subsection{Study 1: Evaluating Generalization of Neural Network}

In the first part, several neural networks were evaluated with different configurations in order to choose the one that showed better results in terms of investment performance. The aim of this study is to find out which neural network configuration provides better results for CAST. Different neural networks configuration schemes were tested and their output was compared with real values in order to choose the one that reached better prediction rates. Next table shows the results from all neural networks tested: 
Table 4. Results of neural networks

\begin{tabular}{|lccll|}
\hline Name & $\begin{array}{c}\text { \# Input } \\
\text { Parms }\end{array}$ & Parms & Test Ok & M-Test Ok (5 VOC) \\
\hline RN1 & 32 & AV, RSI[5-35] & $7.881 \%$ & $65.517 \%$ \\
\hline RN2 & 2 & AV & $8.867 \%$ & $75.862 \%$ \\
\hline RN3 & 5 & AV, RSI $(9,14,30)$ & $11.084 \%$ & $80.296 \%$ \\
\hline RN4 & 32 & AV, RSI[5-35] & $3.941 \%$ & $37.931 \%$ \\
\hline RN5 & 2 & AV & $7.882 \%$ & $63.054 \%$ \\
\hline RN6 & 5 & AV, RSI $(9,14,30)$ & $7.389 \%$ & $53.695 \%$ \\
\hline
\end{tabular}

Results show that RN3 configuration provides best results from the set analyzed. Using M-Test Column, results show that RN3 configuration can predict $80 \%(652$ cases from 812) in an accurate way, which is a very good approach. With the objective of verifying if results presented statistical significant differences among neural networks configurations, the statistical method analysis of variance (ANOVA) was used to carry out analysis of variance among groups using the tool SPSS. The level of statistical significance was set at 0.05. It was used One-way ANOVA in order to test for differences among two or more independent groups (in our case six groups). The results of the test indicate that groups present significant differences indicated by the statistical value $(\mathrm{F}(811)=89.704, \mathrm{p}<.05)$. This circumstance implies that, from a statistical point of view, there is a difference among predictions. However, it is important to work out if there's a difference between best prediction configuration and real values. It was done using the statistical method Student's t-test (comparison of two means). The results of this test showed significant differences between real values and best predictions corresponding to $\mathrm{RN} 3(\mathrm{t}(811)=20.716, \mathrm{p}<.05)$. This circumstance reveals that there are ways to improve our solution either by better training or new setup definition, although it presents undeniable good results. In order to proceed with Study 2 the neural network that will be used as reference for further studies will be the one named RNAR3 for its remarkable capability of generalization according to its results.

\subsection{Study 2: Evaluating Behavior of Neural Network in a Real Case}

In Study 2, once chosen in Study 1 the best neural network configuration (RN3), it was tested applying a real market scenario in a given period of time. The aim of this study is to know if iRSI proposed in this paper gives better results than conventional RSI using 14 days as was suggested by Wilder. In Study 2 this method will be noted by RSI14. Given that CAST can be applied to two different scenarios, in the one hand whole market prediction (IBEX 35, in this case), and on the other hand, single company prediction (pertaining IBEX 35), there will be two main tests. The aim of the first test is to compare iRSI versus RSI14 for IBEX 35 stock market. The aim of the second test is to prove that iRSI can predict single company values pertaining IBEX 35 in a more accurate manner compared with RSI14. The comparison method, which is common to test 1 and test 2, is as follows: given a concrete day and IBEX 35 index (test 1) or Company (test 2), the index is performed (RSI14 and iRSI). It advises either "Sell" or "Buy". Once the action is performed the value related to this operation (of selling or buying) is compared with the final value after a period of time (from one 
day to seven days). If the value is coherent with the prediction (lower if a sell command was send or higher if it was delivered a buy command) then this action increments a success counter for the method proved, RSI14 in this case. Final score will be (success operations) / (total operations).

The sample used in this test consisted in the data obtained in the period between the 16th December 2005 and the 27th October 2009, a total of 812 values. For Test 1 , IBEX 35 values were used. For Test 2, fifteen companies of Spanish stock market (IBEX35) were used using 812 values per company, a total of 12,180 values.

\section{TEST 1}

Next table shows the results of the chosen neural network for iRSI prediction compared with RSI14 predicting IBEX 35 behavior:

Table 5. RSI14 vs iRSI

\begin{tabular}{|c|c|c|}
\hline Period & RSI14 & iRSI \\
\hline 1 Day & $45.65 \%$ & $\mathbf{5 3 . 8 3 \%}$ \\
\hline 2 Days & $43.00 \%$ & $55.46 \%$ \\
\hline 3 Days & $43.48 \%$ & $56.15 \%$ \\
\hline 4 Days & $42.27 \%$ & $\mathbf{5 8 . 4 7 \%}$ \\
\hline 5 Days & $41.79 \%$ & $58.61 \%$ \\
\hline 6 Days & $41.79 \%$ & $58.61 \%$ \\
\hline 7 Days & $42.27 \%$ & $58.20 \%$ \\
\hline
\end{tabular}

The results obtained show a better performance of iRSI compared to RSI14. All measures present higher values in iRSI and all of them are above 50\%. On the other hand, none of the measures by RSI14 present measures above $46 \%$. Higher success values for iRSI are present in 5 and 6 days period $(58.61 \%)$. The first test performed in order to find out if there are significant differences between RSI14 and iRSI was a Student's t-test (comparison of two means). The test was performed for the predictions done without taking into account periods. The results of this test showed significant differences between iRSI and RSI14 $(\mathrm{t}(11367)=15.407, \mathrm{p}<.05)$. This circumstance that can be easily inferred from results reveals that iRSI is a better guidance for investors in order to predict IBEX35 stock market. Applying this same test to pairs of predictions, results show in every case a better prediction as well as a significant difference between every RSI14 and iRSI pair. The second issue is to find out, on the one hand, if there are significant differences among periods as a whole in iRSI. To do so, it was used One-way ANOVA in order to test for differences among two or more independent groups (in this case seven groups). The result of this test pointed out that there are no significant differences among periods for $\operatorname{iRSI}(\mathrm{F}(5683)=1.236, \mathrm{p}>.05)$. This asseveration can be inferred also from results, showing just slight differences among scores.

The application of neural networks to Ibex35 stock prediction is not new [21]. In these cases, neural networks provide a reasonable description of asset price movements, in our case, in which the aim is to improve RSI, results show an unquestionable empowerment of results compared with RSI14. 


\section{TEST 2}

Finally, we show the results of the prediction using the neural network (RNAR3) adapted with the heuristic of RSI calculus using some companies of the IBEX35 market. Table 6 shows results of the application of RSI14 for selected enterprises pertaining IBEX 35 stock market:

Table 6. Results of RSI14 to selected enterprises

\begin{tabular}{|l|llllllllll|} 
Company & \multicolumn{1}{l}{$\begin{array}{l}\text { RSI14 } \\
\text { 1D }\end{array}$} & $\begin{array}{l}\text { RSI14 } \\
\text { 2D }\end{array}$ & PR & \multicolumn{1}{l}{$\begin{array}{l}\text { RSI14 } \\
\text { 3D }\end{array}$} & $\begin{array}{l}\text { PSI14 } \\
\text { 4D }\end{array}$ & $\begin{array}{l}\text { PSI14 } \\
\text { 5D }\end{array}$ & $\begin{array}{l}\text { RSI14 } \\
\text { 6D }\end{array}$ & $\begin{array}{l}\text { PSI14 } \\
\text { 7D }\end{array}$ \\
\hline Enagas & $52.36 \%$ & $56.22 \%$ & $54.51 \%$ & $56.22 \%$ & $57.51 \%$ & $57.94 \%$ & $55.36 \%$ \\
\hline ACS & $43.60 \%$ & $46.71 \%$ & $44.29 \%$ & $41.18 \%$ & $41.52 \%$ & $41.18 \%$ & $42.21 \%$ \\
\hline Inditex & $49.78 \%$ & $48.89 \%$ & $49.33 \%$ & $49.78 \%$ & $48.00 \%$ & $45.33 \%$ & $43.11 \%$ \\
\hline Telecinco & $44.30 \%$ & $44.73 \%$ & $45.15 \%$ & $45.15 \%$ & $40.93 \%$ & $41.77 \%$ & $41.77 \%$ \\
\hline Santander & $49.57 \%$ & $50.00 \%$ & $53.02 \%$ & $55.60 \%$ & $53.02 \%$ & $50.43 \%$ & $50.43 \%$ \\
\hline Indra & $58.38 \%$ & $59.46 \%$ & $62.70 \%$ & $63.78 \%$ & $65.95 \%$ & $65.41 \%$ & $65.41 \%$ \\
\hline Abengoa & $52.40 \%$ & $50.92 \%$ & $48.71 \%$ & $50.18 \%$ & $47.97 \%$ & $49.45 \%$ & $51.29 \%$ \\
\hline Iberia & $48.28 \%$ & $49.43 \%$ & $42.53 \%$ & $43.30 \%$ & $38.70 \%$ & $41.38 \%$ & $38.31 \%$ \\
\hline Iberdrola & $49.29 \%$ & $46.79 \%$ & $46.79 \%$ & $49.29 \%$ & $47.14 \%$ & $44.64 \%$ & $45.71 \%$ \\
\hline Repsol & $48.24 \%$ & $51.37 \%$ & $52.16 \%$ & $50.98 \%$ & $50.20 \%$ & $53.33 \%$ & $52.55 \%$ \\
\hline Sacyr & $48.37 \%$ & $46.88 \%$ & $49.85 \%$ & $48.66 \%$ & $45.10 \%$ & $44.81 \%$ & $43.03 \%$ \\
\hline BBVA & $47.58 \%$ & $50.40 \%$ & $50.81 \%$ & $52.02 \%$ & $49.60 \%$ & $51.21 \%$ & $50.00 \%$ \\
\hline Banesto & $42.97 \%$ & $46.39 \%$ & $45.25 \%$ & $42.21 \%$ & $41.06 \%$ & $38.02 \%$ & $39.92 \%$ \\
\hline Telefónica & $49.09 \%$ & $48.18 \%$ & $47.73 \%$ & $45.91 \%$ & $46.82 \%$ & $46.82 \%$ & $46.82 \%$ \\
\hline Abertis & $55.90 \%$ & $52.31 \%$ & $52.31 \%$ & $49.74 \%$ & $50.26 \%$ & $49.74 \%$ & $47.69 \%$ \\
\hline
\end{tabular}

Next table shows results of the application of iRSI using RNAR3. Figures in bold mean better results for iRSI than RSI14.

Table 7. Results of iRSI using RNA3

\begin{tabular}{|lll|lllll|}
\hline Company & RSI14 & RSI14 & RSI14 & RSI14 & RSI14 & RSI14 & RSI14 \\
PR 1D & PR 2D & PR 3D & PR 4D & PR 5D & PR 6D & PR 7D \\
\hline Enagas & $46.49 \%$ & $48.43 \%$ & $49.93 \%$ & $48.43 \%$ & $48.58 \%$ & $48.43 \%$ & $47.83 \%$ \\
\hline ACS & $\mathbf{4 7 . 3 1 \%}$ & $\mathbf{5 0 . 7 5 \%}$ & $\mathbf{4 9 . 8 5 \%}$ & $\mathbf{5 0 . 9 0 \%}$ & $\mathbf{5 1 . 7 9 \%}$ & $\mathbf{5 1 . 4 9 \%}$ & $\mathbf{5 1 . 1 9 \%}$ \\
\hline Inditex & $\mathbf{5 0 . 3 0 \%}$ & $\mathbf{5 2 . 9 9 \%}$ & $\mathbf{5 4 . 3 4 \%}$ & $\mathbf{5 4 . 9 4 \%}$ & $\mathbf{5 3 . 2 9 \%}$ & $\mathbf{5 2 . 1 0 \%}$ & $\mathbf{5 4 . 5 7 \%}$ \\
\hline Telecinco & $\mathbf{4 9 . 4 1 \%}$ & $\mathbf{5 4 . 4 5 \%}$ & $\mathbf{5 5 . 4 9 \%}$ & $\mathbf{5 6 . 6 8 \%}$ & $\mathbf{5 6 . 9 7 \%}$ & $\mathbf{5 6 . 8 2 \%}$ & $\mathbf{5 6 . 2 3 \%}$ \\
\hline Santander & $\mathbf{5 1 . 4 9 \%}$ & $\mathbf{5 2 . 9 9 \%}$ & $\mathbf{5 4 . 4 8 \%}$ & $55.52 \%$ & $\mathbf{5 5 . 3 7 \%}$ & $\mathbf{5 5 . 5 2 \%}$ & $\mathbf{5 5 . 9 7 \%}$ \\
\hline Indra & $48.49 \%$ & $53.02 \%$ & $54.38 \%$ & $55.89 \%$ & $56.19 \%$ & $55.44 \%$ & $55.29 \%$ \\
\hline Abengoa & $\mathbf{5 3 . 9 3 \%}$ & $\mathbf{5 3 . 7 8 \%}$ & $\mathbf{5 2 . 1 5 \%}$ & $\mathbf{5 2 . 5 9 \%}$ & $\mathbf{5 3 . 3 3 \%}$ & $\mathbf{5 2 . 1 5 \%}$ & $\mathbf{5 2 . 7 4 \%}$ \\
\hline Iberia & $\mathbf{4 9 . 9 3 \%}$ & $\mathbf{4 9 . 9 3 \%}$ & $\mathbf{4 8 . 1 4 \%}$ & $\mathbf{5 0 . 0 7 \%}$ & $\mathbf{4 9 . 4 8 \%}$ & $\mathbf{5 1 . 1 2 \%}$ & $\mathbf{5 1 . 5 6 \%}$ \\
\hline Iberdrola & $\mathbf{5 3 . 0 6 \%}$ & $\mathbf{5 2 . 6 1 \%}$ & $\mathbf{5 3 . 5 0 \%}$ & $\mathbf{5 3 . 5 0 \%}$ & $\mathbf{5 4 . 2 5 \%}$ & $\mathbf{5 2 . 6 1 \%}$ & $\mathbf{5 3 . 3 5 \%}$ \\
\hline Repsol & $\mathbf{5 6 . 5 4 \%}$ & $\mathbf{5 3 . 8 3 \%}$ & $50.38 \%$ & $\mathbf{5 2 . 3 3 \%}$ & $\mathbf{5 2 . 7 8 \%}$ & $51.43 \%$ & $52.48 \%$ \\
\hline Sacyr & $\mathbf{5 5 . 4 4 \%}$ & $\mathbf{5 5 . 1 4 \%}$ & $\mathbf{5 5 . 1 4 \%}$ & $\mathbf{5 6 . 8 0 \%}$ & $\mathbf{5 7 . 4 0 \%}$ & $\mathbf{5 7 . 8 5 \%}$ & $\mathbf{5 9 . 6 7 \%}$ \\
\hline BBVA & $\mathbf{5 4 . 4 0 \%}$ & $\mathbf{5 2 . 9 1 \%}$ & $\mathbf{5 4 . 9 9 \%}$ & $\mathbf{5 4 . 9 9 \%}$ & $\mathbf{5 4 . 5 5 \%}$ & $\mathbf{5 4 . 5 5 \%}$ & $\mathbf{5 4 . 4 0 \%}$ \\
\hline Banesto & $\mathbf{5 2 . 0 2 \%}$ & $\mathbf{5 3 . 3 6 \%}$ & $\mathbf{5 3 . 6 6 \%}$ & $\mathbf{5 4 . 4 1 \%}$ & $\mathbf{5 5 . 4 6 \%}$ & $\mathbf{5 4 . 2 6 \%}$ & $\mathbf{5 5 . 3 1 \%}$ \\
\hline Telefónica & $48.80 \%$ & $\mathbf{4 9 . 1 0 \%}$ & $\mathbf{4 9 . 4 0 \%}$ & $\mathbf{4 8 . 0 5 \%}$ & $\mathbf{4 7 . 4 6 \%}$ & $\mathbf{4 7 . 4 6 \%}$ & $\mathbf{4 7 . 9 0 \%}$ \\
\hline Abertis & $52.82 \%$ & $51.48 \%$ & $51.63 \%$ & $\mathbf{5 3 . 4 1 \%}$ & $\mathbf{5 3 . 5 6 \%}$ & $\mathbf{5 4 . 4 5 \%}$ & $\mathbf{5 4 . 3 8 \%}$ \\
\hline
\end{tabular}


As it can be observed in the result section of the evaluation the use of the neural networks using heuristic formulas calculated by linear regression of financial factors for training can improve RSI14.

\section{Conclusions and Future Work}

The current work describes a research work about the generation of RSI values to create systems capable of generate automated or semi-automated investments on certain companies in the IBEX35 Spanish stock market. In this paper the main work is based in the study case of generate a heuristic for a concrete market, and apply some corrections factor in order to be able to generate good investment results for a concrete companies of the sector. This work was only based in RSI financial indicator and the heuristic methods applied where generated to create a single heuristic formula for IBEX35 stock market. The current work proposes four types of initiatives which should be explored in future research. In the first place, our future work plans to generate heuristic for each company analyzing their data. In the second place, extend the application of iRSI to a broader sample: more companies pertaining IBEX 35, more indexes both national and international and, of course, as stated before, more time frame. In the third place, tune iRSI to adapt it to a momentum in the market. Finally, expand the research to investigate a broader technical analysis indexes like MACD (Moving Average Convergence / Divergence) financial indicator or Bollinger Bands.

Acknowledgments. This work is supported by the Spanish Ministry of Industry, Tourism, and Commerce under the EUREKA project SITIO (TSI-020400-2009-148), SONAR2 (TSI-020100-2008-665 and GO2 (TSI-020400-2009-127). Furthermore, this work is supported by the General Council of Superior Technological Education of Mexico (DGEST). Additionally, this work is sponsored by the National Council of Science and Technology (CONACYT) and the Public Education Secretary (SEP) through PROMEP.

\section{References}

1. Wen, Q., Yang, Z., Song, Y., Jia, P.: Automatic stock decision support system based on box theory and SVM algorithm. Expert Systems with Applications 37(2), 1015-1022 (2010)

2. Wang, Y.F.: Mining stock prices using fuzzy rough set system. Expert System with Applications 24(1), 13-23 (2003)

3. Chiu, D.Y., Chen, P.J.: Dynamically exploring internal mechanism of stock market by fuzzy-based support vector machines with high dimension input space and genetic algorithm. Expert Systems with Applications 36(4), 1240-1248 (2009)

4. Hiemstra, C., Jones, D.: Testing for Linear and Nonlinear Granger Causality in the Stock Price-volume Relation. Journal of Finance 49(5), 1639-1664 (1994)

5. Black, A.J., Mcmillan, D.G.: Non-linear predictability of value and growth stocks and economic activity. Journal of Business Finance and Accounting 31(3/4), 439-474 (2004)

6. Bao, D., Yang, Z.: Intelligent stock trading system by turning point confirming and probabilistic reasoning. Expert Systems with Applications 34(1), 620-627 (2008) 
7. Chang, P.C., Liu, C.H.: A TSK type fuzzy rule based system for stock price prediction. Expert Systems with Applications 34(1), 135-144 (2008)

8. Leigh, W., Modani, N., Purvis, R., Roberts, T.: Stock market trading rule discovery using technical charting heuristics. Expert Systems with Applications 23(2), 155-159 (2002)

9. Edwards, R., Magee, J.: Technical analysis of stock trends, 7th edn. Amacom, New York (1997)

10. Malkiel, B.G.: A random walk down wall street. Norton \& Co., New York (1995)

11. Wang, J.L., Chan, S.H.: Stock market trading rule discovery using two-layer bias decision tree. Expert Systems with Applications 30(4), 605-611 (2006)

12. Kovalerchuk, B., Vityaev, E.: Data mining in finance: advances in relational and hybrid methods. Kluwer Academic, Dordrecht (2000)

13. White, H.: Economic prediction using neural networks: The case of IBM daily stock returns. In: Proceedings of the 2nd Annual IEEE Conference on Neural Networks, II, pp. 451-458 (1988)

14. Kimoto, T., Asakawa, K., Yoda, M., Takeoka, M.: Stock market prediction system with modular neural network. In: Proceedings of the International Joint Conference on Neural Networks, San Diego, CA, pp. 1-6 (1990)

15. Trippi, R.R., DeSieno, D.: Trading equity index futures with a neural network. Journal of Portfolio Management 19(1), 27-33 (1992)

16. Aiken, M., Bsat, M.: Forecasting market trends with neural networks. Information Systems Management 6(4), 42-48 (1994)

17. Wang, L.P., Fu, X.J.: Data Mining with Computational Intelligence. Springer, Heidelberg (2005)

18. Yao, J., Herbert, J.P.: Financial time-series analysis with rough sets. Applied Soft Computing 9(3), 1000-1007 (2009)

19. Wilder Jr., J.W.: New Concepts in Technical Trading Systems. Hunter Publishing Company, Greensboro (1978)

20. Arulampalam, G., Bouzerdoum, A.: A generalized feedforward neural network architecture for classification and regression. Neural Networks 16(5-6), 561-568 (2003)

21. Fernández-Rodríguez, F., González-Martel, C., Sosvilla-Rivero, S.: On the profitability of technical trading rules based on artificial neural networks: Evidence from the Madrid stock market. Economics Letters 69(1), 89-94 (2000) 